\title{
Assessment of the Willingness to Collaborate in Enterprise Networks
}

\author{
João Rosas and Luis M. Camarinha-Matos \\ Universidade Nova de Lisboa, Faculty of Sciences and Technology, \\ 2829-516 Monte Caparica, Portugal \\ \{jrosas, cam\}@uninova.pt
}

\begin{abstract}
The success of a partnership depends fundamentally on the partners' active and vigorous participation in the achievement of the common goals. The commitment to these goals is associated to each partner's attitudes and intentions. These intentions, in turn, are linked to the partner's beliefs concerning the expected outcomes of a collaboration opportunity. This work proposes an approach to assess organizations' willingness to collaborate. This approach follows a behavioral perspective, which is based on the Theory of the Planned Behavior. A tool named Intentions Query Mechanism selects a number of appropriate questions from a knowledge-base, from which the answers allow to estimate the level of an organization's willingness to collaborate.
\end{abstract}

Keywords: Organization's behavior, willingness to collaboration, collaborative networks, Theory of the Planned Behavior.

\section{Introduction}

Willingness to collaborate in a networked organization is related to the partner's attitudes and intentions towards concrete collaboration situations. It is dependent on a variety of aspects, which are basically related to the expected outcomes of a collaboration opportunity, such as increased profits, achieving new markets, or obtaining some competitive advantages. When a partner perceives a collaboration opportunity as beneficial, its intention or willingness to collaborate increases, otherwise it decreases. Other aspects which account for willingness to collaborate are the perceived risks, external incentives, or the presence of a fierce competition.

This work proposes an approach to assess organizations' willingness to collaborate. It follows a behavioral perspective, based on the Theory of the Planned Behavior [1]. The approach involves the assessment of an organization's attitudes towards collaborating, the external influences, or social incentive/pressure to collaborate, and the perceived control over factors that might facilitate/impede the partner's ability to collaborate. Assessing the willingness to collaborate helps to foresee how well an organization is likely to perform in a partnership.

\section{Contribution to Technological Innovation}

In spite of its value, this subject has not been well explored yet. An innovative aspect of this work is that it bases the notion of collaboration willingness on the idea of 
behavioral beliefs, attitudes and intentions concerning collaboration opportunities. Additionally, a systematic approach for collaboration willingness assessment, with automatic inquiring selection, is also proposed. This concept of collaboration willingness is part of a larger collaboration readiness model [2], as illustrated in Fig. 1.

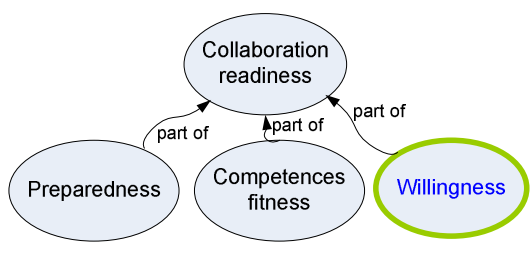

Fig. 1. The collaboration readiness concept

Each component of the readiness model is characterized as follows:

- Preparedness - is based on the concept of organization's character. It aims at assessing whether an organization is likely to display reliable behavior inside partnerships [3]. The greater this likelihood, the greater the preparedness level.

- Competences fitness - This concept is seen as a soft versus hard competencies dichotomy. Hard competencies are associated to the achievement of concrete or "physical" outcomes, while soft competencies are more associated to more abstract or intangible ones. Competencies fitness aims at assessing how well an organization uses its hard competencies in a partnership. Their effective use might also require the exercise of certain soft competencies, such as the ability to share knowledge [4].

- Willingness - It corresponds to the topic of this paper, and aims at assessing partners' attitudes and intentions to participate in a collaboration opportunity.

Similarly to willingness, an innovative aspect of this collaboration readiness model is that it assumes a behavioral perspective aiming at assess partners readiness to collaborate, helping to increase the likelihood of partnerships success. The establishment of this conceptual basis is fundamental for the development of a new generation of tools to support partner's selection for collaborative networks.

\section{Related Research}

At first sight, willingness to collaborate should have taken much attention by collaborative networks researchers, given its potential importance for partnerships success. As a matter of fact, a more generic problem, which can be referred as willingness to perform a particular behavior is already a common subject in human behavior studies [5]. However, such is not the case for the area of collaborative networks, as research works addressing organizations' willingness to collaborate are scarce. An example of such a research is [6] where willingness to collaborate is defined as a new communication trait. As such, willingness to collaborate is positively related to a number of traits, such as willingness to communicate, argumentativeness, verbal aggressiveness, 
interpersonal communication competency, and a relaxed, friendly and attentive communicator style. In [7] it is analyzed the influence of organization's corporate culture, and perceived culture of a particular partner, in order to undertake a shift from a transactional buyer-seller relationship to an intensified collaboration. If there is fitness between own and peer's culture, then willingness to cooperate increases.

Other works could be mentioned here, but are not related to collaboration. The research from [8] is concerned with a firm's willingness to engage in innovation. The research in [9], addresses a firms' willingness to adopt cleaner technologies.

The topic of willingness to collaborate seems to be an unexplored subject in the field of collaborative networks.

\section{Modeling Willingness to Collaborate}

As mentioned before, willingness to collaborate is concerned with assessing a partner's attitudes and intentions towards concrete collaboration situations. A theory that seems to be useful here is the Theory of the Planned Behavior (TPB) proposed by Ajzen [1]. Therefore, before introducing the proposed approach, a brief introduction to $T P B$ is made.

The Theory of the Planned Behavior. Many works from social sciences aiming at predicting people's behavior are based on the Theory of Planned Behavior. This theory aims at predicting the people's willingness to engage in a particular behavior. According to this theory, human action is guided by three kinds of considerations:

- Behavioral beliefs, which correspond to beliefs about the likely outcomes from performing such behavior, and the evaluation of these outcomes.

- Normative beliefs, which correspond to beliefs about the normative expectations of the surrounding environment (other people, the group, or society) and the motivation to comply with these expectations.

- Control beliefs, which correspond to beliefs about the presence of factors that may facilitate or impede the performance of the behavior, and the perceived power of these factors.

In their respective aggregates, the behavioral beliefs produce a favorable or unfavorable attitude towards the behavior in question; normative beliefs result in perceived social pressure or subjective norm towards performing the behavior; and control beliefs give rise to perceived behavioral control. In combination, these factors lead to the formation of a behavioral intention. According to TPB, this intention is assumed to be the immediate antecedent or predictor of the behavior [1].

The adopted assumption is that $T P B$ can also be considered suitable for predicting the organizations' behaviors. This suitability lies in the assumption that organizations behave like rational agents, making systematic use of the available information, in order to decide which goals to pursuit. These goals are achieved through corresponding business processes, usually established at a strategic level. Assuming that organization's strategic planning is based on goals to be achieved suggests that these goals can be seen as organizations' intentions to perform the corresponding behaviors. In other words, they correspond to the organizations' planned behavior [8]. As a result, 
if an organization's intentions are based on goals to be achieved, and if organization's behaviors are in turn a consequence of these intentions, then the TPB can be applied to organizations in order to predict their behavior. Consequently, TPB can be used to assess organizations' willingness to collaborate.

The collaboration willingness model. The assessment of the willingness to collaborate depends on the perception of an organization's intentions concerning a particular collaboration opportunity $(\mathrm{CO})$ and other factors, named as background factors, which are illustrated in Fig. 2. These intentions in turn depend on the organization's attitudes, social influence or incentives, and the perceived control over the collaboration opportunity and these factors. The proposed model for willingness to collaborate follows a structure that is compliant with TPB.

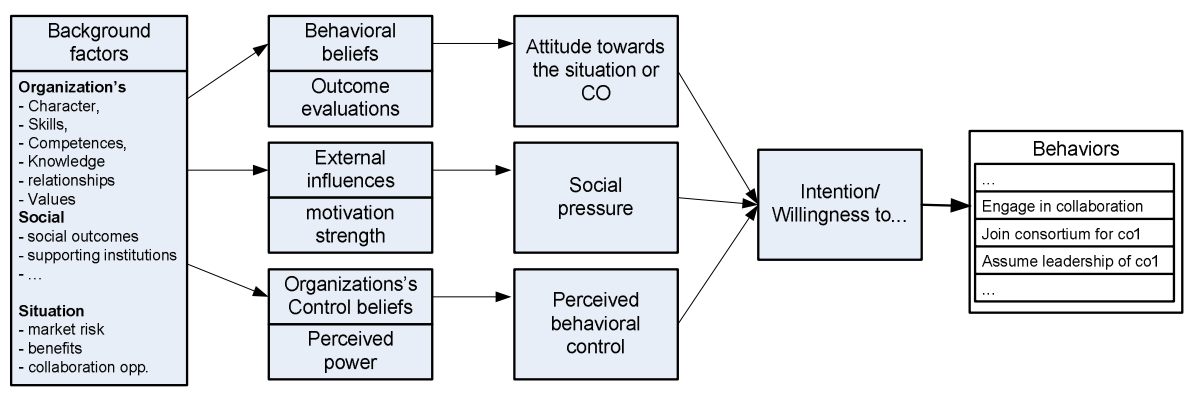

Fig. 2. Willingness to collaborate model

In order to keep the presented model as generic as the TPB in the definitions below, we consider not only the "willingness to collaborate" behavior, but also other behaviors related to collaboration, as illustrated on the right side of Fig. 2. Additionally, in order to illustrate the definitions below, some examples of queries are presented near each definition. These examples aim at assessing an organization's willingness to "engage in a partnership".

Definition 1 (Background factors) - Are the aspects relevant to the perception of an organization's intentions to collaborate in a specific collaboration opportunity.

Definition 2 (Organization's Behavioral beliefs) - Correspond to beliefs that a behavior (e.g. engaging in a partnership) will provide a number of expected outcomes (e.g. higher profit or the access to an extended market). Each behavioral belief is specified by a subjective probability that the corresponding outcome will be achieved. It can be specified as a set of tuples $\left\{\left(b b_{\mathrm{i}}, v_{\mathrm{i}}\right) \mid b b_{\mathrm{i}} \in B B, v_{\mathrm{i}} \in[0,1]\right\}$, in which:

- $\quad b b_{\mathrm{i}}$ - identifies a belief about an outcome from the behavior in question.

- $\quad v_{\mathrm{i}}$ - corresponds to a belief strength, or subjective probability, of the corresponding outcome being achieved.

- $B B=\left\{b b_{1}, b b_{2}, \ldots\right\}-$ represents the set of identified behavioral beliefs concerning collaboration behaviors. 
The organization believes that engaging in the partnership will allow growing abroad. Extremely unlikely

\begin{tabular}{|l|l|l|l|l|l|l|}
\hline & & & & $\mathrm{X}$ & & \\
\hline
\end{tabular}

Extremely likely

Definition 3 (Outcome appraisal) - It represents how an organization values the outcome associated to each behavioral belief in Definition 2. It can be specified as a set $\left\{\left(o a_{\mathrm{i}}, v_{\mathrm{i}}\right) \mid o a_{\mathrm{i}} \in O A, v_{\mathrm{i}} \in[0,1]\right\}$, in which for each tuple:

- $\quad o a_{\mathrm{i}},-$ specifies the outcome associated to the behavioral belief $b b_{\mathrm{i}}$ in Definition 2.

- $\quad v_{\mathrm{i}}$ - represents a subjective appraisal of the expected outcome.

- $O A=\left\{o a_{1}, o a_{2}, \ldots\right\}-$ represents the set of outcomes, each related one-by-one to a behavioral belief specified in Definition 2 .

The organization thinks growing abroad is Not important

\begin{tabular}{|l|l|l|l|l|l|l|}
\hline & & & & $\mathrm{X}$ & & \\
\hline 1 & 2 & 3 & 4 & 5 & 6 & 7 \\
\hline
\end{tabular}

Very important

Definition 4 (Attitude towards a behavior) - It is the degree ' $A_{b}$ ' to which the performance of a behavior $b$ is positively or negatively valuated by the organization. This value is determined as a composite of the behavioral beliefs (Definition 2) and corresponding evaluations (Definition 3). Specifically, the strength of each belief $b b_{\mathrm{i}}$ is weighted by the appraisal $o a_{\mathrm{i}}$ of the outcome, and then aggregated using the following equation:

$$
A_{b}=\frac{1}{n \times(\# s c a l e)^{2}} \times \sum_{i=1}^{n} \pi_{2}\left[\left(b b_{i}, v_{i}\right)\right] \times \pi_{2}\left[\left(o a_{i}, v_{i}\right)\right]
$$

The first part of the above equation, and for the next ones, transforms the result into a percentage format. The symbol (\#scale) stands for the size of the used scale. For instance, if the scale is defined as scale $=\{1,2,3,4,5,6,7\}$, then (\#scale) $=7$. Additionally, $\pi_{2}$ stands for the algebraic projection, which retrieves the second element of the corresponding tuple given as argument.

Definition 5 (External stimulus or influences) - Are used to represent external influences, which favor or disfavor the performance of the behavior in question, such as engaging in a partnership. It can be specified as a set $\left\{\left(e s_{\mathrm{i}}, v_{\mathrm{i}}\right) \mid e s_{\mathrm{i}} \in E S, v_{\mathrm{i}} \in\right.$ $[0,1]\}$, in which for each tuple:

- $\quad e s_{\mathrm{i}}-$ identifies an external stimulus for the behavior in question

- $v_{\mathrm{i}}$ - specifies the degree of belief in that stimulus.

- $E S=\left\{e s_{1}, e s_{2}, \ldots\right\}-$ represents the external stimulus or influences, which potentially influence the organization in the performance of the considered behavior.

Will the organization receive support for investing abroad?

Not likely

\begin{tabular}{|l|l|l|l|l|l|l|}
\hline & & $\mathrm{X}$ & & & & \\
\hline 1 & 2 & 3 & 4 & 5 & 6 & 7 \\
\hline
\end{tabular}

Quite likely 
Definition 6 (Motivation to comply) - Establishes the degree or strength of motivation to which an organization complies with the external influences (Definition 5) over the performance of the behavior. It can be specified as a set $\left\{\left(m c_{\mathrm{i}}, v_{\mathrm{i}}\right) \mid m c_{\mathrm{i}} \in\right.$ $\left.M C, v_{\mathrm{i}} \in[0,1]\right\}$, in which for each tuple:

- $\quad m c_{\mathrm{i}}$ - identifies a motivation to comply with the external stimulus $e s_{\mathrm{i}}$ specified in Definition 5.

- $\quad v_{\mathrm{i}}$ - Its corresponding motivation strength.

- $M C=\left\{m c_{1}, m c_{2}, \ldots\right\}-$ The motivators associated to the performance of a behavior.

How important is receiving support to invest aboard for this organization?

Not important

\begin{tabular}{|l|l|l|l|l|l|l|}
\hline & & & & & $X$ & \\
1 & 2 & 3 & 4 & 5 & 6 & 7
\end{tabular} Very important

Definition 7 (Social influence) - It corresponds to the composite aggregation of each external stimulus with the corresponding motivations to comply. Specifically, the strength of each external stimulus $e s_{i}$ is weighted by motivation to comply $m c_{i}$, using the following equation:

$$
S I_{b}=\frac{1}{n \times(\# \text { scale })^{2}} \sum_{i=1}^{n} \pi_{2}\left[\left(e s_{i}, v_{i}\right)\right] \times \pi_{2}\left[\left(m c_{i}, v_{i}\right)\right]
$$

Definition 8 (Organization's control beliefs) - It is concerned with the beliefs an organization has got on the factors that may facilitate or impede the performance of the behavior. It can be specified as a set $\left\{\left(c b_{\mathrm{i}}, v_{\mathrm{i}}\right) \mid c b_{\mathrm{i}} \in C B, v_{\mathrm{i}} \in[0,1]\right\}$, in which for each tuple:

- $\quad c b_{\mathrm{i}}$ - identifies a control factor that may facilitate, or impede, the performance of the behavior.

- $\quad v_{\mathrm{i}}$ - the subjective probability that the corresponding factor is present.

- $C B=\left\{c b_{1}, c b_{2}, \ldots\right\}-$ The identified control beliefs set associated to the performance of the behavior.

Is the organization prepared to operate abroad?

Not likely

\begin{tabular}{|l|l|l|l|l|l|l|}
\hline & & & $\mathrm{X}$ & & & \\
\hline 1 & 2 & 3 & 4 & 5 & 6 & 7 \\
\hline
\end{tabular}

Quite likely

Definition 9 (Perceived power) - Corresponds to the perceived value of each control factor, as specified in Definition 8. It can be specified as a set $\left\{\left(p p_{\mathrm{i}}, v_{\mathrm{i}}\right) \mid p p_{\mathrm{i}} \in P P, v_{\mathrm{i}}\right.$ $\in[0,1]\}$, in which for each tuple:

- $\quad p p_{\mathrm{i}}-$ identifies the perceived power for the control factor in the corresponding control belief $c b_{\mathrm{i}}$ specified in Definition 8 .

- $v_{\mathrm{i}}-$ the value for the corresponding $p p_{\mathrm{i}}$.

- $\quad P P=\left\{p p_{1}, p p_{2}, \ldots\right\}-$ The identified perceived power set associated to the performance of the behavior. 


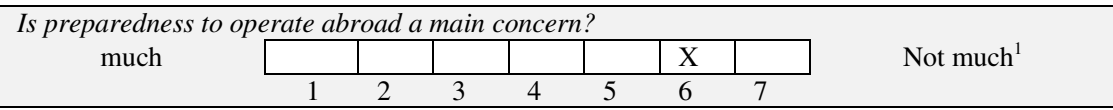

Definition 10 (Perceived behavioral control) - It refers to the organization's perceptions on its capability to perform the behavior. This element is determined by the aggregation of the control beliefs with the perceived power of each one. Specifically, the strength of each control belief $c b_{\mathrm{i}}$ is weighted by the perceived power $p p_{\mathrm{i}}$, and the products are aggregated using the following equation:

$$
P B C_{b}=\frac{1}{n \times(\# \text { scale })^{2}} \sum_{i=1}^{n} \pi_{2}\left[\left(c b_{i}, v_{i}\right)\right] \times \pi_{2}\left[\left(p p_{i}, v_{i}\right)\right]
$$

Definition 11 (Organization's willingness to collaborate) - According to TPB, intention to collaborate can be taken as a direct measure of the willingness to collaborate. Intention is based on the attitude toward the behavior (Definition 4), the social influences (Definition 7), and perceived behavioral control (Definition 10). Each of these factors is weighted or adjusted taking into consideration the behavior or situations. Its value can be computed by a function

$$
\begin{gathered}
I=F(A T B, S I, P B C)=w_{1} * A T B+w_{2} * S I+w_{3} * P B C . \\
W \sim I
\end{gathered}
$$

The weights $w_{1}, w_{2}$ and $w_{3}$ represent correlation coefficients. They indicate to which extent the values of $A T B, S I$ and $P B C$ influence the willingness to collaborate level. Their determination requires a separated pilot study, in which a questionnaire sent to organizations, which participated in previous partnerships, would allow determine the correlation between these values and the the willingness to collaborate level. This determination is typically made using correlation or regression analysis.

The values of attitude $A_{\mathrm{b}}$, social influence $S B_{\mathrm{b}}$ and perceived behavioral control $P C B_{\mathrm{b}}$ for the provided examples can be obtained, using the above definitions, as following:

$$
\left\{\begin{array}{c}
A_{\text {engage in the partnership }}=\frac{1}{1 \times 49} \times 4 \times 5=0.41 \\
S I_{\text {engage in the partnership }}=\frac{1}{1 \times 49} \times 3 \times 6=0.37 \\
P C B_{\text {engage in the partnership }}=\frac{1}{1 \times 49} \times 4 \times 6=0.49
\end{array}\right.
$$

Assuming the weight values as $w_{1}=0.33, w_{2}=0.33$ and $w_{3}=0.33$, the level of the willingness to collaborate is

$$
W \sim I_{\text {engage in the partnership }}=0.33 *(0.41+0.37+0.49)=42 \%
$$

The Intentions Query Mechanism. In order to provide a systematic way of assessing the willingness to collaborate, a tool named Intentions Query Mechanism (IQM) was

\footnotetext{
${ }^{1}$ The scale used in this example is inverted to ensure that the right side always reflects a positive attitude.
} 
developed. From the description of a collaboration opportunity, this tool selects the a set of possible questions to be asked, in order to assess willingness to collaborate. The approach used to develop the IQM is based on a Prolog knowledge-base, which manages an experts' repository of questions. These questions are used to enquire partners' attitudes towards collaboration-related behaviors and expected outcomes. From the text description of a given collaboration opportunity, the tool detects the semantic relationships between the terms used in the $\mathrm{CO}$ description and the questions stored in the repository. Each time the tool finds a match, the corresponding term is highlighted, as illustrated in Fig. 3. The discovery of the semantic relationships is performed with using WordNet, a large network of semantic relations between English words [10]. An example of a semantic relationship is between the words "listen" and "perceive", for which WordNet stores the fact that "to perceive" is a hypernym of "to listen".

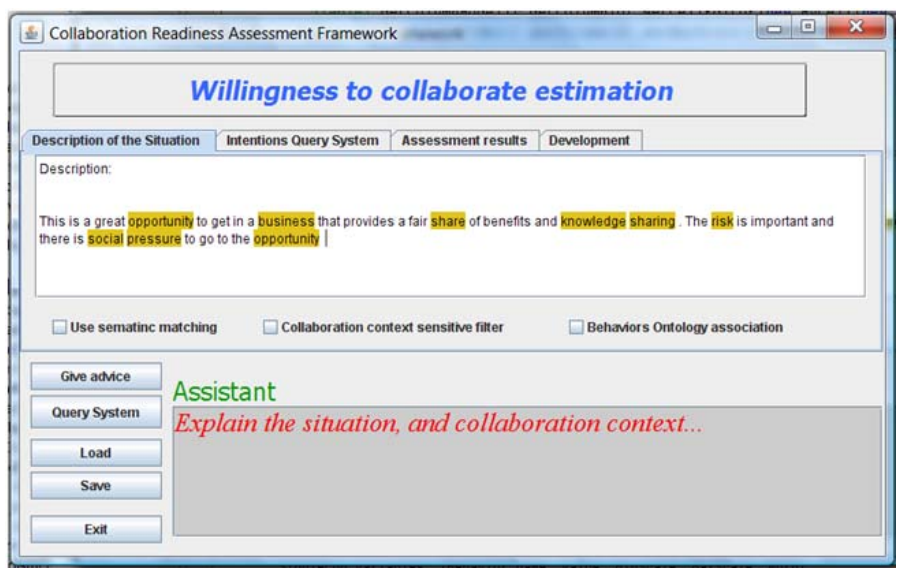

Fig. 3. Describing the collaboration opportunity

The highlighted terms are subsequently used to select the specific questions from the knowledge-base, which is presented to a concerned user for the necessary answers, as illustrated in

Fig. 4. These questions are specified according to the definitions presented in previous section. As they aim at evaluating subjective behavioral beliefs, the corresponding answers do not require strict crisp values.

Finally, these values are provided as input to the TPB part and the result is a number, which represents the organization's willingness level to collaborate, as specified in Definition 11. Using this definition, the willingness to collaborate level for the organization in the example is $50.96 \%$, as shown in Fig. 5. An interpretation of this result for a concrete organization could be that this organization's willingness to engage in a given collaboration opportunity is not appreciably high. 


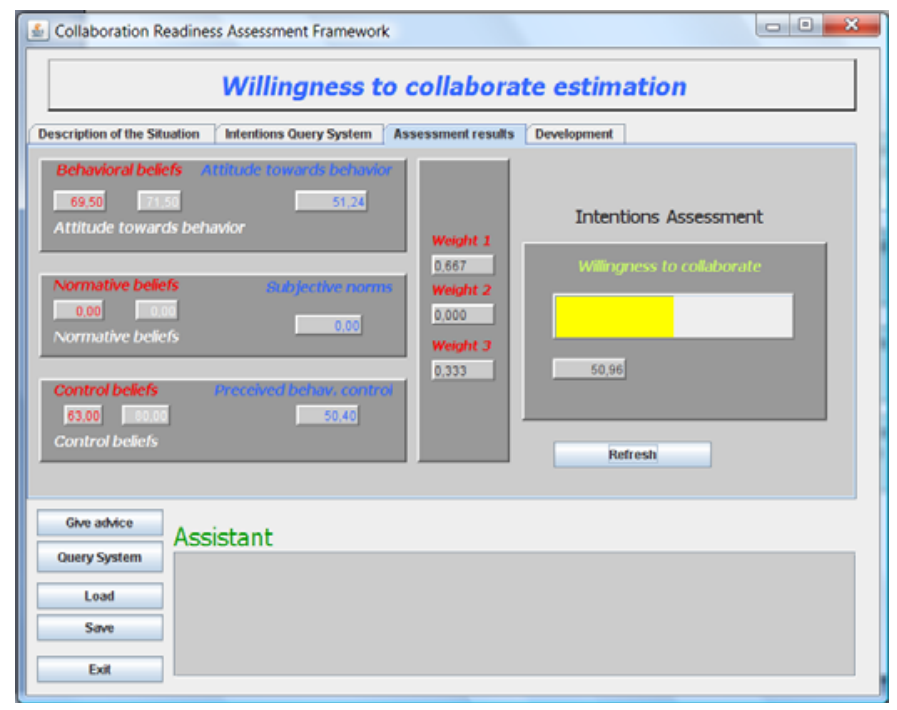

Fig. 4. Estimation of willingness to collaborate

\section{Conclusions and Future Work}

In spite of the importance for collaborative networks, research works concerning willingness to collaborate are scarce, particularly those following a behavioral perspective. This work formalized this concept of collaboration willingness, and introduced a corresponding assessment approach, based on the Theory of the Planned Behavior. A tool named Intentions Query Mechanism provides a way to systematically assess this willingness to collaborate.

The preliminary results show that this assessment approach is feasible and promising. Nevertheless, further research is needed to improve it into a more complete assessment model.

Acknowledgments. This work was supported in part by the Portuguese "Fundação para a Ciência e a Tecnologia" through PhD scholarship.

\section{References}

1. Ajzen, I.: The theory of planned behavior. Organizational behavior and human decision processes 50, 179-211 (1991)

2. Rosas, J., Camarinha-Matos, L.M.: An approach to assess collaboration readiness. International Journal of Production Research 47, 4711-4735 (2009)

3. Rosas, J., Camarinha-Matos, L.M.: Modeling collaboration preparedness assessment. In: Collaborative Networks: Reference Modeling. Springer, US (2008)

4. Rosas, J., Macedo, M., Camarinha-Matos, L.M.: An Organization's extended (Soft) Competencies Model. In: Camarinha-Matos, L.M., Paraskakis, I., Afsarmanesh, H. (eds.) PRO-VE 2009. IFIP AICT, vol. 307. Springer, Heidelberg (2009) 
5. Ajzen, I.: From intentions to actions: A theory of planned behavior. In: Kuhl, J., Beckmann, J. (eds.) Action control: From cognition to behavior, pp. 11-39. Springer, New York (1985)

6. Anderson, C.M., Martin, M.M., Infante, D.A.: Decision-making collaboration scale: Tests of validity. Communication Research Reports 15, 245-255 (1998)

7. Claudia, K., Michael, L.: Philipps: Corporate culture and its impact on the willingness to cooperate in the distribution channel: conceptualization and empirical finding in the German hospital industry. University of Marburg

8. Corral, C.M.: Explaining and predicting the innovative behaviour of the firm: a behavioural approach

9. Montalvo Corral, C.: Sustainable production and consumption systems-cooperation for change: assessing and simulating the willingness of the firm to adopt/develop cleaner technologies. The case of the In-Bond industry in northern Mexico. Journal of cleaner production 11, 411-426 (2003)

10. George, A.M.: WordNet: a lexical database for English. Commun. ACM 38, 39-41 (1995) 